\title{
New Layout Strategies with Improved Matching Performance
}

\author{
Chengming $\mathrm{He}^{1}$, Xin $\mathrm{Dai}^{2}$, Hanqing Xing ${ }^{2}$, and Degang Chen ${ }^{2}$ \\ ${ }^{1}$ Silicon Laboratories, Inc., Austin, TX 78735 \\ ${ }^{2} \mathrm{ECpE}$, Iowa State University, Ames, IA 50011
}

\section{Abstract}

In this paper, the systematic mismatch error in integrated circuits due to gradient effects is modeled and analyzed. Three layout strategies with improved matching performance are reviewed and summarized. The hexagonal tessellation pattern can cancel quadratic gradient errors with only 3 units for each device and has high area-efficiency when extended. Both the $N^{\text {th }}$-order circular symmetry patterns and $N^{\text {th }}$-order central symmetry patterns can cancel up to $N^{\text {th }}$-order gradient effects between two devices using $2^{N}$ unit cells for each one. Among these three techniques, the central symmetry patterns have the best-reported matching performance for Manhattan structures; the circular-symmetry patterns have the best theoretical matching performance; and the hexagonal tessellation pattern has high density and high structural stability. The $N^{\text {th }}$-order central symmetry technique is compatible to all IC fabrication processes requiring no special design rules. Simulation results of these proposed techniques show better matching characteristics than other existing layout techniques under nonlinear gradient effects. Specifically, two pairs of P-poly resistors using $2^{\text {nd }}$ and $3^{\text {rd }}$-order central symmetry patterns were fabricated and tested. Less than $0.04 \%$ mismatch and less than $0.002 \%$ mismatch were achieved for the $2^{\text {nd }}$ and the $3^{\text {rd }}$-order structures, respectively.

Key words: matching, symmetry, layout, and pattern 


\section{INTRODUCTION}

In VLSI circuits, mismatch errors are the difference between two or more device parameters that are desired to be identical. Matching accuracy, to some extent, dominates the performance of analog and mixed-signal integrated circuits. For example, matching of sampling capacitors in switched-capacitor (SC) circuits directly affects the performance of pipelined/cyclic ADCs and SC filters. Matching characteristics of current mirrors play a key role in many applications $[1,2]$. In modern communications circuits such as quadrature modulators, I/Q matching directly affects the image-rejection ratio, which is a key performance index. Matching in a differential amplifier limits reduction of evenorder harmonics, especially the $2^{\text {nd }}$ order harmonic. Layout techniques to handle mismatch errors become more important to high-performance circuits design, since even a small amount of mismatch may easily hurt the performance of a precision circuit.

Over the years, great efforts have been made to the study of mismatch and layout strategies [3-6]. Previous studies show that the causes of mismatch can be categorized as systematic and random variations. The random variations are usually modeled by zero mean Gaussian distribution and tradeoffs can be made between area and matching accuracy [4]. The systematic variations are process dependent and usually modeled as spatial gradients in device parameters. The mismatch due to systematic variations may be at the same level of that of random variations [7]. If the random mismatch is reduced by increasing the area, the systematic mismatch becomes dominant. Furthermore, increasing area actually make the gradient effect more significant. Since mismatch due to systematic variations can cause performance degradation, it should be carefully handled and minimized. 
Despite the widely recognized importance of matching, existing design and layout strategies dealing with the systematic mismatch are quite limited. Putting unit cells closely to each other reduces the gradient effect, but does not cancel it. The widely used common centroid layout pattern can only compensate for linear gradient [8]. Although the fully differential structure is robust to even-order harmonics, $2^{\text {nd }}$ order gradients can introduce $3^{\text {rd }}$-order harmonics that fully differential structure cannot reduce. This error limits the dynamic range of some precision circuits. In this paper, three layout techniques capable of canceling mismatch errors due to high-order gradient effects are introduced. The $N^{\text {th }}$-order circular symmetry [6] and $N^{\text {th }}$-order central symmetry patterns [9] can cancel mismatch errors introduced by linear to $N^{\text {th }}$-order gradient effects, when each device uses $2^{N}$ unit cells. The hexagonal tessellation pattern [6] can cancel quadratic gradient effect with only 3 units for each device and has high area-efficiency. Among these three layout techniques, central symmetry patterns have the best matching performance for Manhattan structures; circular-symmetry patterns have the best theoretical matching performance; and the hexagonal tessellation pattern has high density and high structural stability with its honeycomb structure. The $N^{\text {th }}$-order central symmetry technique is compatible to all IC fabrication processes requiring no special design rules. These properties are proven by theoretical derivation and their matching performance is evaluated using MATLAB simulation.

The rest of the paper is organized as follows. In Section II, a general mathematical model of gradient effects is given. Section III describes three layout strategies and shows how they can cancel nonlinear gradient effects. Section IV gives the simulation results of the proposed layout strategies and some measurement results. 


\section{GRADIENT MODELING}

A two dimensional polynomial function $p(x, y)$ can be used to model a parameter at the point $(x, y)$. A parameter that has linear gradient can be modeled as

$$
p_{1}(x, y)=G_{1}(x, y)+C,
$$

where $(x, y)$ is the coordinate of the point of interest, $C$ is a constant, and

$$
G_{1}(x, y)=g_{1,0} x+g_{0,1} y
$$

is the linear gradient component of $p . g_{1,0}$ and $g_{0,1}$ are the linear gradient coefficients. Equation (1) can be easily extended to higher-order cases. Generally, a parameter that has up to $n^{\text {th }}$-order gradient components can be modeled as

$$
p_{n}(x, y)=\sum_{j=1}^{n} G_{j}(x, y)+C,
$$

where $\quad G_{j}(x, y)=\sum_{k=0}^{j} g_{k, j-k} x^{k} y^{j-k}$

is the $j^{\text {th }}$-order component. $g_{k, j-k}$ 's are the $j^{\text {th }}$-order coefficients.

Now consider one of the unit cells composing a device, the parameter of the unit cell is the integral of the parameter value over the area of the unit cell. Since the area of the unit cell is usually small, the gradient effect over the unit cell is negligible and the parameter of the unit cell can be approximated by the parameter at a particular point $\mathrm{P}$ in the unit cell. Using the location of this point as the location of the unit cell, for a device composed of $m$ unit cells located at $\left(x_{1}, y_{1}\right) \ldots\left(x_{m}, y_{m}\right)$, we can get the device's parameter as 


$$
P=\sum_{i=1}^{m} p_{n}\left(x_{i}, y_{i}\right)
$$

where $n$ is the highest order of the gradient effect. For two devices A and B, ideal matching is achieved if the mismatch error

$$
\Omega(A, B)=P_{A}-P_{B}=0 .
$$

Substituting $x$ with $\left(x-x_{0}+x_{0}\right)$ and y with $\left(y-y_{0}+y_{0}\right)$ in (3), we get

$$
p_{n}(x, y)=\sum_{k=1}^{n} \sum_{j=0}^{k} g_{j, k-j}\left(x-x_{0}+x_{0}\right)^{j}\left(y-y_{0}+y_{0}\right)^{k-j}+C
$$

which can be rewritten as

$$
\begin{aligned}
& p_{n}(x, y)=\sum_{j=0}^{n} g_{j, n-j}\left(x-x_{0}+x_{0}\right)^{j}\left(y-y_{0}+y_{0}\right)^{n-j}+ \\
& \sum_{k=1}^{n-1} \sum_{j=0}^{k} g_{j, k-j}\left(x-x_{0}+x_{0}\right)^{j}\left(y-y_{0}+y_{0}\right)^{k-j}+C
\end{aligned}
$$

Defining the $1^{\text {st }}$ item to be $I_{1}$ and expending it gives

$$
I_{1}=\sum_{j=0}^{n} g_{j, n-j}\left(\left(\sum_{k=0}^{j}\left(\begin{array}{l}
j \\
k
\end{array}\right) x_{0}^{j-k}\left(x-x_{0}\right)^{k}\right) \times\left(\sum_{l=0}^{n-j}\left(\begin{array}{l}
n-j \\
l
\end{array}\right) y_{0}^{n-j-l}\left(y-y_{0}\right)^{l}\right)\right) .
$$

Equation (9) can be rewritten as

$$
I_{1}=\sum_{j=0}^{n} g_{j, n-j}\left(x-x_{0}\right)^{j}\left(y-y_{0}\right)^{n-j}+\sum_{j=0}^{n} g_{j, n-j} \sum_{k \geq 0, l \geq 0, k+l \leq n-1} a_{k, l}\left(x-x_{0}\right)^{k}\left(y-y_{0}\right)^{l}
$$

where $\alpha_{k, l}$ is the coefficient of $\left(x-x_{0}\right)^{k}\left(y-y_{0}\right)^{l}$ assuming $x_{0}$ and $y_{0}$ constant. Notice that the order of the $2^{\text {nd }}$ term in (8) and the $2^{\text {nd }}$ term in (10) are both no greater than $(n-1)$. That means (8) can be expressed in the form of 
$p_{n}(x, y)=\sum_{j=0}^{n} g_{j, n-j}\left(x-x_{0}\right)^{j}\left(y-y_{0}\right)^{n-j}+\sum_{i=1}^{n-1} G_{i}^{\prime}(x, y)+C$

where $\quad G_{i}^{\prime}(x, y)=\sum_{j=0}^{i} g_{j, i-j}^{\prime} x^{j} y^{i-j}$

has the same form as $G_{i}(x, y)$, but with different coefficients. And $C^{\prime}$ is a constant. Equation (11) shows that the center of $n^{\text {th }}$ order gradient can be moved from $(0,0)$ where $G_{n}(x, y)=0$ to arbitrarily any point $\left(x_{0}, y_{0}\right)$ so that $G_{n}\left(x-x_{0}, y-y_{0}\right)=0$, and this will only introduce lower order gradient components.

\section{THREE LAYOUT TECHNIQUES CANCELLING HIGH-ORDER NONLINEAR GRADIENT}

\section{1 $N^{\text {th }}$-order central symmetry pattern}

The central symmetrical layout pattern is for 1-1 matching between two devices. A description of the pattern is as follows:

i) The $1^{\text {st }}$ order form of the pattern is just any common centroid pattern. Such as shown in Fig. 1(a) and (b). Common centroid layout pattern ensures the cancellation of linear $\left(1^{\text {st }}\right.$ order) gradient error.

ii) The $n^{\text {th }}(n>1)$ order central symmetrical pattern can be defined in terms of the $(n-1)^{\text {st }}$ order pattern. The $\mathrm{n}^{\text {th }}$ order pattern is composed of two $n-1^{\text {st }}$ order patterns symmetrical to a center $C_{\mathrm{n}}$. There are two cases according to n's parity:

a) If $\mathrm{n}$ is odd, the unit cells of each device is central symmetrical around $C_{\mathrm{n}}$. That means for each unit cell of device $\mathrm{A}$ at point $\mathrm{P}$, there is another unit cell of device $\mathrm{A}$ at point $\mathrm{P}$ ' and the middle point of segment PP' is exactly the symmetrical center $C_{\mathrm{n}}$. 
b) If $\mathrm{n}$ is even, the unit cells of the two devices in one of the $\mathrm{n}-1^{\text {st }}$ order patterns should be interchanged so that the position of device A's unit cells are central symmetrical to device B's unit cells around $C_{\mathrm{n}}$. That means for each unit cell of device $\mathrm{A}$ at point $\mathrm{P}$, there is an unit cell of device $\mathrm{B}$ at point $\mathrm{P}^{\prime}$ and the middle point of segment $\mathrm{PP}^{\prime}$ is exactly the symmetrical center $C_{\mathrm{n}}$. Fig. 2 and Fig. 3 show some high order (n>=2) central symmetrical layout patterns.

The following analysis will show how the central symmetrical layout pattern can cancel nonlinear gradient effect. Suppose both device A and device B has $m$ unit cells.

i) If $n=1$, the parameter only has linear gradient effect. According to (5), the parameter of device $\mathrm{A}$ is

$$
P_{A}=\sum_{i=1}^{m} p_{1}\left(x_{A i}, y_{A i}\right)=\sum_{i=1}^{m}\left(g_{1,0} x_{A i}+g_{0,1} y_{A i}+C\right)
$$

Similarly, the parameter of device B is

$$
\begin{aligned}
& P_{B}=\sum_{i=1}^{m} p_{1}\left(x_{B i}, y_{B i}\right)=\sum_{i=1}^{m}\left(g_{1,0} x_{B i}+g_{0,1} y_{B i}+C\right) \\
& \begin{array}{|l|l|l|l|l|}
\hline \mathbf{A} & \mathbf{B} & \mathbf{B} & \mathbf{A} & \mathbf{B} \\
\text { (a) } & & \mathbf{B} & \mathbf{A} \\
\hline \text { (b) }
\end{array}
\end{aligned}
$$

Fig. 1 Examples of $1^{\text {st }}$ order central symmetrical pattern

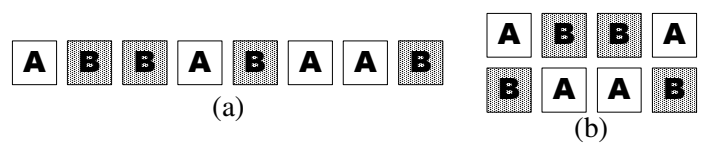

Fig. 2 Examples of $2^{\text {nd }}$ order central symmetrical pattern 


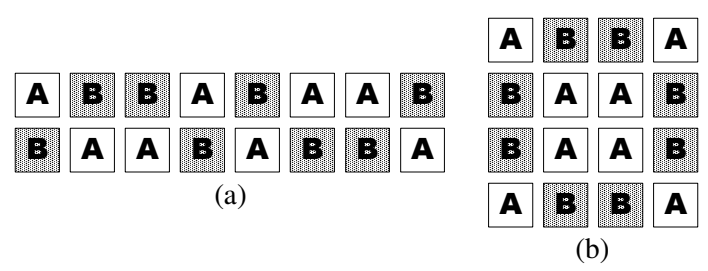

Fig. 3 Examples of $3^{\text {rd }}$ order central symmetrical pattern

The centroid of a device composed of $m$ unit cells located at $\left(x_{i}, y_{i}\right), i=1,2, \ldots, m$ are defined as $\left(x_{c}, y_{c}\right)$ where

$$
\begin{aligned}
& x_{c}=\frac{1}{m} \sum_{i=1}^{m} x_{i} \\
& y_{c}=\frac{1}{m} \sum_{i=1}^{m} y_{i}
\end{aligned}
$$

From (13) (16), it is not difficult to derive that (6) holds if and only if $x_{C A}=x_{C B}$ and $y_{c A}=y_{c B}$. This is why common centroid layout pattern can cancel linear gradient effect.

ii) Assume our proposed $n^{\text {th }}$-order central symmetry layout can cancel mismatch from linear to the $(n-1)^{\text {th }}$-order nonlinear gradient. Then if $n>1$, since the higher order pattern are constructed by duplicating lower order patterns, the number of unit cells of each device, $m$, must be an even number. Now consider the two cases according to n's parity

\section{If $n$ is odd}

Consider device $\mathrm{A}$, according to the layout pattern, for a unit cell $\mathrm{A}_{i}$ at $\left(x_{A i}, y_{A i}\right)$, there must be another unit cell $\mathrm{A}_{m-i}$ at $\left(x_{A m-i}, y_{A m-i}\right)$ which meets $x_{A i}-x_{C n}=x_{C n}-x_{A m-i}$ and $y_{A i}-y_{C n}=y_{C n}-y_{A m-i}$. Then for any $0 \leq j \leq n$,

$\left(x_{A i}-x_{C n}\right)^{j}\left(y_{A i}-y_{C n}\right)^{n-j}=-\left(x_{A, m-i}-x_{C n}\right)^{j}\left(y_{A, m-i}-y_{C n}\right)^{n-j}$. 
Choosing $x_{0}$ and $y_{0}$ in (11) to be $x_{C n}$ and $y_{C n}$ and substituting (11) to (5) gives

$$
P_{A}=\sum_{i=1}^{m} \sum_{j=1}^{n-1}\left(G_{j}^{\prime}\left(x_{A i}, y_{A i}\right)+C^{\prime}\right)
$$

Since unit cells of device B have the same central symmetry property, we get

$$
P_{B}=\sum_{i=1}^{m} \sum_{j=1}^{n-1}\left(G_{j}^{\prime}\left(x_{B i}, y_{B i}\right)+C^{\prime}\right)
$$

Equations (18) and (19) give

$$
P_{A}-P_{B}=\sum_{i=1}^{m} \sum_{j=1}^{n-1}\left(G_{j}^{\prime}\left(x_{A i}, y_{A i}\right)-G_{j}^{\prime}\left(x_{B i}, y_{B i}\right)\right)
$$

This means the mismatch due to the $\mathrm{n}^{\text {th }}$ order gradient effect has been cancelled.

\section{If $n$ is even}

According to the layout pattern, for an A's unit cell $\mathrm{A}_{i}$ at $\left(x_{A i}, y_{A i}\right)$, there is a B's unit cell $\mathrm{B}_{i}$ at $\left(x_{B i}, y_{B i}\right)$ which meets $x_{A i}-x_{C \mathrm{n}}=x_{C \mathrm{n}}-x_{B i}$ and $y_{A i}-y_{C \mathrm{n}}=y_{C \mathrm{n}}-y_{B i}$. Then

$$
\left(x_{A i}-x_{C n}\right)^{j}\left(y_{A i}-y_{C n}\right)^{n-j}=\left(x_{B i}-x_{C n}\right)^{j}\left(y_{B i}-y_{C n}\right)^{n-j}
$$

Choosing $x_{0}$ and $y_{0}$ in (11) to be $x_{C n}$ and $y_{C n}$, and then substitute (11) to (5) gives

$$
P_{A}=\sum_{i=1}^{m}\left(\sum_{j=0}^{n} g_{j, n-j}\left(x_{A i}-x_{C n}\right)^{j}\left(y_{A i}-y_{C n}\right)^{n-j}+\sum_{j=1}^{n-1}\left(G_{j}^{\prime}\left(x_{A i}, y_{A i}\right)+C^{\prime}\right)\right.
$$

and

$$
P_{B}=\sum_{i=1}^{m}\left(\sum_{j=0}^{n} g_{j, n-j}\left(x_{B i}-x_{C n}\right)^{j}\left(y_{B i}-y_{C n}\right)^{n-j}+\sum_{j=1}^{n-1}\left(G_{j}^{\prime}\left(x_{B i}, y_{B i}\right)+C^{\prime}\right) .\right.
$$

Subtracting (23) from (22) still results in (20). 
So the mismatch due to the $\mathrm{n}^{\text {th }}$ order gradient effect is cancelled for any $\mathrm{n}>1$. Since the $\mathrm{n}^{\text {th }}$ order layout pattern is built from the $\mathrm{n}-1^{\text {st }}$ order layout pattern, which can cancel the first to the $(n-1)^{\text {th }}$ order gradient effect, the $\mathrm{n}^{\text {th }}$ order pattern should preserve this property and thus capable of canceling from the linear to the $\mathrm{n}^{\text {th }}$ order gradient.

Following this induction approach, it is proven that the $\mathrm{N}^{\text {th }}$-order central symmetry layout technique can cancel from $1^{\text {st }}$ up to $\mathrm{n}^{\text {th }}$ order gradient effect.

\section{$3.2 N^{\text {th }}$ order circular symmetry pattern}

The circular symmetry layout pattern is initially proposed for 1-1 matching between two devices. However, theoretical analysis suggests this layout pattern is capable for multiple devices' matching. A description of the pattern is as follows (each unit cell is modeled with a single point):

A desired device is composed with $2^{\mathrm{n}}$ unit cells and their centers are located on a circle with an arbitrary center $\left(x_{0}, y_{0}\right)$. The coordinates of these centers are defined in

$$
\begin{aligned}
& \left\{X_{i} \mid\left(x_{0}+\rho \cos \theta_{i}, y_{0}+\rho \sin \theta_{i}\right), i=1, \ldots, 2^{n}\right\} \\
& \left\{\theta \mid \theta_{i}=\theta_{0}+(i-1) \frac{2 \pi}{2^{n}}, i=1, \ldots, 2^{n}\right\}
\end{aligned}
$$

Fig. 4 illustrates a second order circular symmetry patterns. The following analysis will show how the circular symmetry layout pattern can cancel nonlinear gradient effect.

Using the gradient model (1)-(4), the effect of gradient on a point $A_{i}$ can be expressed as (26) and the total effect can be expressed by equation (27)

$$
p_{n}\left(A_{i}\right)=p_{n}\left(x_{0}+\rho \cos \theta_{i}, y_{0}+\rho \sin \theta_{i}\right)
$$




$$
P_{A}=\sum_{i=1}^{2^{n}} p_{n}\left(A_{i}\right)
$$

Using trigonometry theory, we can use equation (28) to represent (26) with appropriate coefficients. The coefficients are determined by the coefficients in equations (1)-(4).

$$
p_{n}\left(A_{i}\right)=g_{0}\left(x_{0}, y_{0}, \rho\right)+\sum_{j=1}^{n} g_{j}\left(x_{0}, y_{0}, \rho\right) \sin \left(j \theta_{i}+\phi_{j}\right)
$$

Thus we can extend equation (27) to (29).

$$
\begin{aligned}
& P_{A}=2^{n} g_{0}\left(x_{0}, y_{0}, \rho\right)+\sum_{i=1}^{2 \wedge n} \sum_{j=1}^{n} g_{j}\left(x_{0}, y_{0}, \rho\right) \sin \left(j \theta_{i}+\phi_{j}\right) \\
& =2^{n} g_{0}\left(x_{0}, y_{0}, \rho\right)+\sum_{j=1}^{n} g_{j}\left(x_{0}, y_{0}, \rho\right) \sum_{i=1}^{2 \wedge^{n}} \sin \left(j \theta_{i}+\phi_{j}\right)
\end{aligned}
$$

Define $v P_{j}=g_{j}\left(x_{0}, y_{0}, \rho\right) \sum_{i=1}^{2 \wedge_{n}} \sin \left(j \theta_{i}+\phi_{j}\right)$

Then

$$
P_{A}=2^{n} g_{0}\left(x_{0}, y_{0}, \rho\right)+\sum_{j=1}^{n} v P_{j}
$$

We will show $v P_{j}=0$ using the well known equality $\sin (\theta+2 k \pi+\pi)+\sin \theta=0$.

Because $\left\{\theta \mid \theta_{i}=\theta_{0}+(i-1) \frac{2 \pi}{2^{n}}, i=1, \ldots, 2^{n}\right\}$, for $j \leq n$,

$$
\sum_{i=1}^{2^{\wedge} n} \sin \left(j \theta_{i}+\phi_{j}\right)=\sum_{i=1}^{2^{\wedge} n} \sin \left[j \theta_{0}+j(i-1) \frac{2 \pi}{2^{n}}+\phi_{j}\right]
$$

It is not apparent that equation (32) is identical to zero. However, if $\mathrm{j}$ is odd, it is easy to show $\theta_{i+2^{n-1}}-\theta_{i}=j \pi, i=1,2, \cdots, 2^{n-1}$. At such a case, $v P_{j}=0$. If $\mathrm{j}$ is even, we can express 
$j=2^{m} k, k$ is odd . It is easy to show that $\theta_{2^{n-m-1}+i}-\theta_{i}=k \pi$.

$\left\{\theta \mid \theta_{i}=\theta_{0}+(i-1) \frac{2 \pi}{2^{n}}, i=1, \ldots, 2^{n}\right\}$ can be grouped to $2^{m}$ exclusive and complete sub sets.

At both cases, $v P_{j}=0$.

Thus,

$$
P_{A}=2^{n} g_{0}\left(x_{0}, y_{0}, \rho\right)
$$

We call the pattern of $A_{i}, i=1,2, \cdots, 2^{n}$ an $n^{\text {th }}$-order circular symmetry pattern. One of the most important properties of this $\mathrm{n}^{\text {th }}$ order circular symmetry pattern is rotationinvariance. This has been shown in our derivations since $\theta_{0}$ can be any value. Mathematically we could place multiple sets of the $\mathrm{n}^{\text {th }}$-order circular symmetry pattern in the same circle. Thus it is capable of achieving matching among multiple devices. Multiple devices' matching property may have significant advantages in certain applications.

Thus, we have demonstrated and proven a layout pattern which will sufficiently cancel mismatch due to linear gradient and up to the $\mathrm{n}^{\text {th }}$ order nonlinear gradient. It may cancel some higher order nonlinear gradient too. Although the derivations are based on pointrepresents, the conclusion can be applied to a region. Construction of the $\mathrm{N}^{\text {th }}$ order circular symmetry patter would be as following:

a) build a unit cell

b) place $2^{n+1}$ unit cells around a center (rotating the unit cell), the angle between adjacent unit cells to the center is $\frac{\pi}{2^{n}}$ 
c) connect the alternative cells together and form two devices. As shown in Fig. 4b, A and $\mathrm{B}$ are matching up to the quadratic gradient.

It is worth to mention the common centroid layout and the $1^{\text {st }}$ order circular symmetry layout pattern. With some derivations, it will be shown that the device parameter would be a function independent of $\rho$. That just confirms the $1^{\text {st }}$ order circular symmetry pattern is a special common-centroid pattern. In other words, the common-centroid layout is an extended version of the first-order circular symmetry layout pattern.

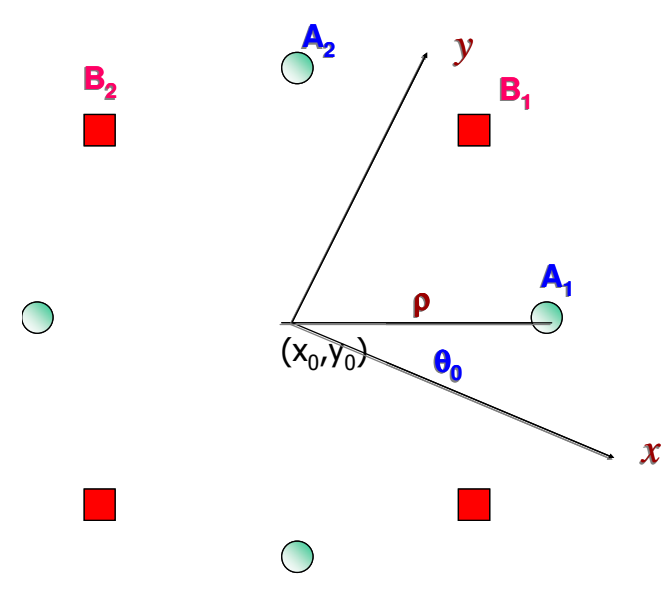

(a)

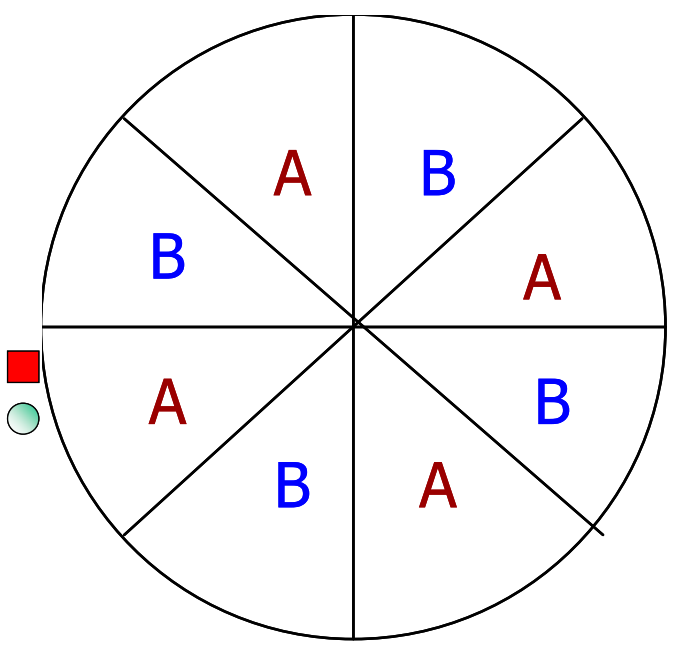

(b)

Fig. $4 \mathrm{~A} 2^{\text {nd }}$-order circular symmetry pattern

\subsection{Hexagonal Tessellation}

Hexagon, the basic cell of bee nest, has wide applications in communication, architecture, chemical engineering and so on because of its high mechanical strength, high spatial efficiency. We will show hexagon also is the most concise layout pattern that can cancel linear and quadratic gradient completely. Furthermore, we can extend hexagon to construct the hexagonal tessellation pattern easily without space-waste. 
Figure 5a shows a hexagon. The coordinates of the six vertexes $\left\{A_{i}, B_{i}, i=1,2,3\right\}$ can be annotated as

$$
\begin{aligned}
& \left\{A_{i}, B_{i} \mid\left(x_{0}+\rho \cos \theta_{i}, y_{0}+\rho \sin \theta_{i}\right), i=1,2,3\right\} \\
& \left\{\theta \mid \theta_{i}=\theta_{0}+(i-1) \frac{2 \pi}{3}+\frac{\pi}{3} a, i=1,2,3\right\} a=1: A ; 0: B
\end{aligned}
$$

For a quadratic gradient ( $\mathrm{n}=2$ in equation 5$)$, we can prove that the total gradient is not related to $\theta_{0}$.

$$
\begin{aligned}
& \sum_{i=1}^{3} p_{2}\left(A_{i}\right)=g\left(x_{0}, y_{0}, \rho\right) \\
& \sum_{i=1}^{3} p_{2}\left(B_{i}\right)=g\left(x_{0}, y_{0}, \rho\right)
\end{aligned}
$$

Thus the gradient effects on both A and B are the same. Mismatch due to linear to quadratic gradient between A and B is cancelled.

Fig. 5b shows a divided hexagon. There are six triangles. Assume each triangle is represented by its center of gravity, the six centers of gravity will form a hexagon in the fashion as that in Fig. 5a. Thus the As and Bs in Fig. 5b match with each other. 


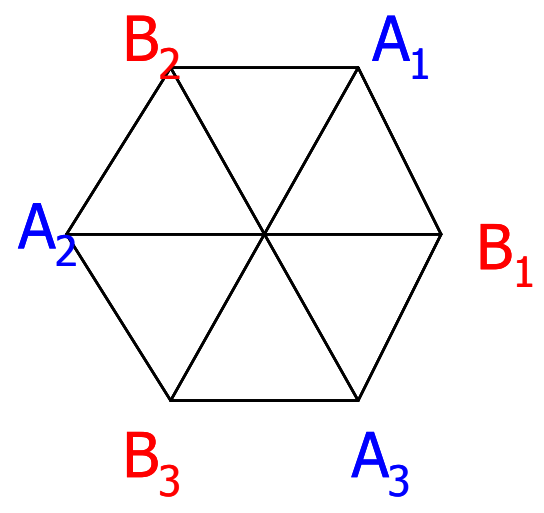

(a)

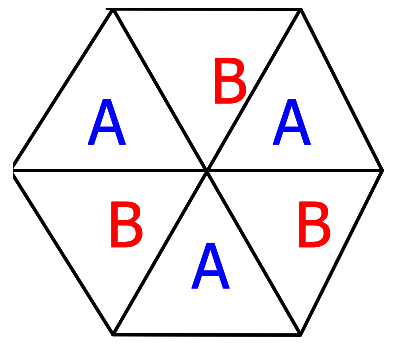

(b)

Fig. 5 Hexagonal matching pattern

As shown in Fig. 6, a hexagon can be extended and filled with As and Bs in a way that forms a honeycomb structure. The layout pattern shown in Fig. 6 is named as hexagon tessellation. Because honeycomb structure is well known for its compact, high areaefficient and low sensitive to stress, the hexagon tessellation pattern would also have excellent matching even under external stress and no area would be wasted.

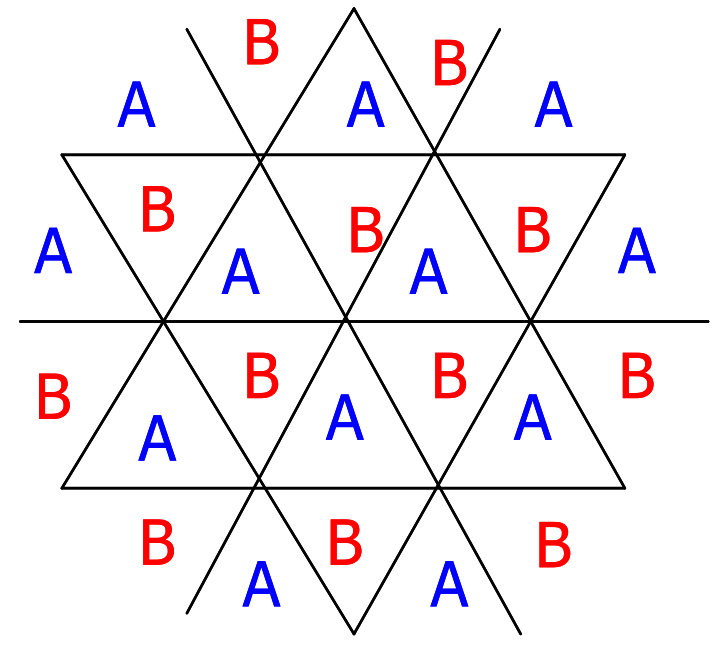

Fig. 6 Hexagonal Tessellation 


\section{EVALUATION OF THESE THREE LAYOUT PATTERNS AND MEASUREMENT RESULTS}

To evaluate the performance of the proposed layout techniques, we did MATLAB simulations on some of the existing layout patterns and the proposed patterns under different gradient effects. The layout patterns we chose are $1^{\text {st }}$ order (common centroid) $5^{\text {th }}$ order central symmetrical pattern (Fig. 7 (a) $\sim($ e) $), 2^{\text {nd }}$ order circular symmetry pattern (Fig. 7(f)) and hexagonal (Fig. 7(g)). Same total device area is assigned for each layout pattern and every unit cell is a rectangle. If triangle unit cell is used, the $2^{\text {nd }}$ order circular symmetry pattern and hexagonal tessellation pattern shown in Fig. 6 should have much better matching performance. Up to $5^{\text {th }}$ order gradient are generated for simulation. When we study the effect of $\mathrm{k}^{\text {th }}$ order gradient, we use $\mathrm{k}^{\text {th }}$-order polynomial terms plus constant 1 .

$$
G_{k}(x, y)=1+a \sum_{i=0}^{k} x^{i} y^{k-i}, a<<1
$$

The simulation results are summarized in Table I where mismatch is defined by (38)

$$
\Omega=2 \times\left|\frac{p_{A}-p_{B}}{p_{A}+p_{B}}\right| \times 100 \%
$$

TABLE I. SIMULATION RESULTS OF DIFFERENT LAYOUT PATTERNS

\begin{tabular}{|c|c|c|c|c|c|}
\hline \multirow{2}{*}{ Mismatch (\%) } & \multicolumn{5}{|c|}{ Highest Order of Gradient } \\
\cline { 2 - 6 } & $\mathbf{1}^{\text {st }}$ & $\mathbf{2}^{\text {nd }}$ & $\mathbf{3}^{\text {rd }}$ & $\mathbf{4}^{\text {th }}$ & $\mathbf{5}^{\text {th }}$ \\
\hline Fig. 7 (a) & 0 & 2.77 & 5.22 & 7.43 & 10.39 \\
\hline Fig. 7 (b) & 0 & 0 & 0.24 & 0.87 & 1.70 \\
\hline Fig. 7 (c) & 0 & 0 & 0 & 0.01 & 0.068 \\
\hline Fig. 7 (d) & 0 & 0 & 0 & 0 & 0.0023 \\
\hline
\end{tabular}




\begin{tabular}{|c|c|c|c|c|c|}
\hline \multirow{2}{*}{ Mismatch (\%) } & \multicolumn{5}{|c|}{ Highest Order of Gradient } \\
\cline { 2 - 6 } & $1^{\text {st }}$ & $2^{\text {nd }}$ & $\mathbf{3}^{\text {rd }}$ & $\mathbf{4}^{\text {th }}$ & $\mathbf{5}^{\text {th }}$ \\
\hline Fig. 7 (e) & 0 & 0 & 0 & 0 & 0 \\
\hline Fig. 7 (f) & 0 & 0 & 0 & 0.026 & 0.18 \\
\hline Fig. 7 (g) & 0 & 0 & 0.26 & 0.50 & 2.24 \\
\hline Fig. 4 (b) & 0 & 0 & 0 & 0.017 & 0.12 \\
\hline Fig. 5 (b) & 0 & 0 & 0.17 & 0.32 & 1.48 \\
\hline
\end{tabular}

Simulation results show that for $n=1, \ldots, 5$, the $n^{\text {th }}$ order central symmetrical pattern can cancel up to nth order gradient effect, which is consistent with the previous analysis. Furthermore, the hexagonal is the efficient layout to cancel up to 2 nd order gradient. The 2nd order circular symmetry pattern cancels up to the 3rd order gradient, instead of only the 1 st and 2nd order gradient as mentioned in [6].In this pattern, the placement of the unit cells of a device is central symmetrical around the center of the circle. According to the analysis in section II and III, when $n=3$ is odd, as long as it cancels up to 2 nd order gradient, it would also cancel the 3 rd order gradient. This also implies that the $(2 n)^{\text {th }}$ order circular symmetry pattern would cancel $(2 n+1)^{\text {th }}$-order nonlinear gradient. Compared with these layout techniques, the central symmetrical layout is more area efficient and flexible in cell placement. And it is easy to be extended to high order cases for cancellation of any high order gradient. 


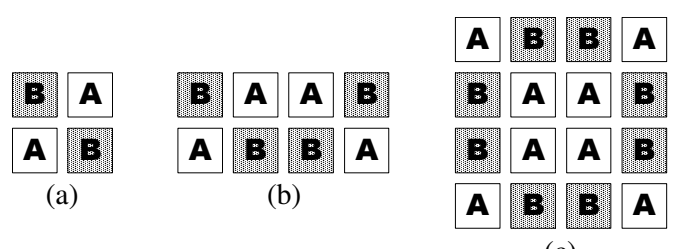

(c)

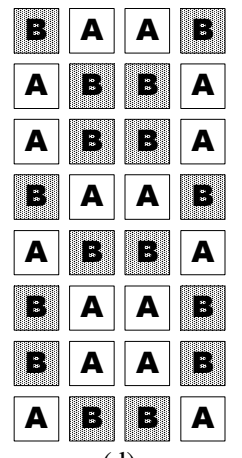

(d)

\begin{tabular}{|c|c|c|c|c|c|c|c|}
\hline $\mathbf{B}$ & $\mathbf{A}$ & $\mathbf{A}$ & $\mathbf{B}$ & $\mathbf{A}$ & $\mathrm{B}$ & B & $\mathbf{A}$ \\
\hline $\mathbf{A}$ & B & B & $\mathbf{A}$ & B & $\mathbf{A}$ & $\mathbf{A}$ & $\mathbf{B}$ \\
\hline $\mathbf{A}$ & $\mathbf{B}$ & B & A & B & $\mathbf{A}$ & $\mathbf{A}$ & B \\
\hline B & A & $\mathbf{A}$ & $\mathbf{B}$ & $\mathbf{A}$ & $\mathrm{B}$ & B & $\mathbf{A}$ \\
\hline $\mathbf{A}$ & B & B & $\mathbf{A}$ & B & $\mathbf{A}$ & $\mathbf{A}$ & $\mathbf{B}$ \\
\hline$B$ & A & $\mathbf{A}$ & $\mathbf{B}$ & A & B & B & $\mathbf{A}$ \\
\hline$B$ & $\mathbf{A}$ & $\mathbf{A}$ & B & A & B & B & $\mathbf{A}$ \\
\hline $\mathbf{A}$ & B & B & $\mathbf{A}$ & B & $\mathbf{A}$ & $\mathbf{A}$ & B \\
\hline
\end{tabular}

(e)
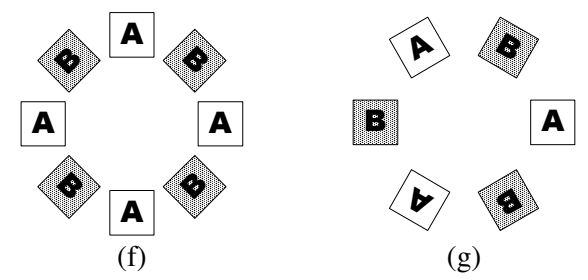

Fig. 7 Layout patterns used in simulation

Our layout strategies do not make use of process information and is independent on causes the gradient errors. Therefore, the experimental results only depend on the actual gradient errors present, but they should be independent of the process used. This is important because process-independent matching property makes our novel layout patterns usable in all available process technologies.

The proposed $\mathrm{N}^{\text {th }}$-order central symmetry pattern has been verified on silicon. Although transistor or capacitor matching might be more interesting, resistors are used in our silicon implementation due to two main reasons. First, the measurement of transistor or capacitor matching is significantly more involved than the measurement of resistance. We want to separate measurement error from gradient-induced mismatch error. Since this paper is the first time the layout strategy is introduced, we wanted to make sure that we were only 
comparing the influence of layout on matching errors and measurement quality can be easily ensured. Second, at the time of designing the circuit prototype at Silicon Labs Inc, one communication circuit required more than $70 \mathrm{~dB}$ image rejection ratio in one pair of resistors. This IRR requires better than $64 \mathrm{~dB}$ matching performance in these two resistors [10]. More product, circuit schematic, layout and fabricated die details could not be disclosed due to the intellectual property concern. For these two reasons, we have selected resistor matching as the first vehicle to demonstrate the new layout strategies. Transistor matching or capacitor matching can be a future study topic. We believe that our analysis, simulations and measurement data clearly demonstrated the effectiveness of improving matching using our proposed new layout strategies.

Based on random mismatch data on TSMC characterization reports, a specific area was allocated to each resistor. The proposed $\mathrm{N}^{\text {th }}$-order central symmetry layout pattern was chosen. In order to reduce complexity of layout work, the $2^{\text {nd }}$-order and $3^{\text {rd }}$-order pattern were adopted and fabricated in 0.13 um CMOS process. For the $2^{\text {nd }}$-order pattern as shown in Fig. 8a, one resistor was constructed by paralleling 4 identical resistor-units. Each had an area of $1 / 8$ of the total area. For the $3^{\text {rd }}$-order pattern shown in Fig. 8 b, each resistor was constructed in a parallel-series connection combining 8 unit cells in the $3^{\text {rd }}$-order central symmetry pattern. Measurements of more than 100 chips show that the $2^{\text {nd }}$-order one has less than $0.04 \%$ systematic mismatch errors and the $3^{\text {rd }}$-order central symmetry pattern has less than $0.002 \%$ systematic mismatch errors. Due to company's intellectual property requirement, other measurement details could not be disclosed. These measurement results confirmed the matching improvement using our proposed high-order nonlinear gradient canceling layout patterns. 


\begin{tabular}{|c|c|c|}
\hline \multicolumn{1}{|c|}{ Dummy } & \multicolumn{2}{|c|}{ Dummy } \\
\hline Resistor A & Resistor A & Resistor B \\
\hline Resistor B & Resistor B & \multicolumn{1}{|c|}{ Resistor A } \\
\hline Resistor B & Resistor B & Resistor A \\
\hline Resistor A & Resistor A & Resistor B \\
\hline Resistor B & Resistor B & Resistor A \\
\hline Resistor A & Resistor A & Resistor B \\
\hline Resistor A & Resistor A & Resistor B \\
\hline Resistor B & Resistor B & Resistor A \\
\hline Dummy & & \multicolumn{2}{|c|}{ Dummy } \\
\hline
\end{tabular}

(a)

(b)

Fig. 8 Two precisely-matched resistor pairs

\section{ConClusions}

This paper modeled and analyzed the systematic mismatch due to linear and nonlinear gradient effects. Based on the analysis, we proposed three layout techniques capable of canceling mismatch errors due to high-order gradient effects. The $\mathrm{N}^{\text {th }}$-order circular symmetry [6] and $\mathrm{N}^{\text {th }}$-order central symmetry patterns [9] can cancel mismatch from linear to the $\mathrm{n}^{\text {th }}$-order gradient between two devices by using $2^{\mathrm{n}}$ unit cells for each one; the hexagonal tessellation pattern [6] can cancel quadratic gradient with only 3 units for each device and has high area-efficiency. Among these three layout techniques, central symmetry patterns have the best reported matching performance for Manhattan structures; circular-symmetry patterns have the best theoretical matching performance; hexagonal tessellation pattern has high density, high structural stability with its honeycomb structure. The $\mathrm{N}^{\text {th }}$-order central symmetry technique is compatible to all IC fabrication processes requiring no special design rules. All layout patterns have been mathematically proved and verified through simulation. Testing results of the proposed $\mathrm{N}^{\text {th }}$-order central symmetry layout pattern confirmed our analysis and simulation. 


\section{References}

[1] V. Gupta, G. A. Rincon-Mora, "Predicting the effects of error sources in bandgap reference circuits and evaluating their design implications," Circuits and Systems, 2002, MWSCAS-2002, vol. 3, pp. 575-578, Aug. 2002.

[2] H. A. Alzaher, M. Ismail, "Robust low-distortion wideband CMOS current-follower," Electronics Letters, vol. 35, issue. 25, pp. 2203-2204, Dec. 1999.

[3] S. Lovett, M. Welten, A. Mathewson, B. Mason, "Optimizing MOS transistor mismatch,” IEEE J. Solid-State Circuits, vol. 33, pp. 147-150, Jan. 1998.

[4] M. J. M. Pelgrom, A. C. J. Duinmaijer, A. P. G. Welbers, "Matching properties of MOS transistors,” IEEE J. Solid-State Circuits, vol. SC-24, pp 1433-1439, 1989.

[5] K. Lakshmikumar, R.Hadaway, and M.Copeland, "Characterization and modeling of mismatch in MOS transistors for precision analog design," IEEE J. Solid-State Circuits, vol. SC-21, pp. 1057-1066, 1986.

[6] Chengming He, Kuangming Yap, Degang Chen, R. Geiger, " $\mathrm{N}^{\text {th }}$ order circular symmetry pattern and hexagonal tesselation: two new layout techniques cancelling nonlinear gradient," Circuits and Systems, Proceedings of the 2004 International Symposium on, vol. 1, pp. 237-240, May 2004.

[7] Eric Felt, "Measurement and Modeling of MOS Transistor Current Mismatch in Analog IC's," Proc. of ACM, pp. 272-277, 1994.

[8] A. Hastings, The Art of Analog Layout. Prentice Hall, New Jersey, 2000.

[9] Xin Dai, Chengming He, Hanqing Xing, Degang Chen, Randall Geiger, " $\mathrm{N}^{\text {th }}$ Order Central Symmetrical Layout Pattern for Nonlinear Gradient Cancellation," Circuits 
and systems, Proceedings of the 2005 International Symposium on, pp.4835-4838, May 2005

[10] Behbahani, F.; Kishigami, Y.; Leete, J.; Abidi, A.A., "CMOS mixers and polyphase filters for large image rejection," IEEE J. Solid-State Circuits, Vol. 36, June 2001 pp. 873-887 
Affiliation of author(s): Chengming He, is currently a design engineer in Silicon Laboratories, Inc.; Xin Dai, Hanqing Xing and Degang Chen are from the department of electrical and computer engineering at Iowa State University. 
Fig. 1 Examples of $1^{\text {st }}$ order central symmetrical pattern

Fig. 2 Examples of $2^{\text {nd }}$ order central symmetrical pattern

Fig. 3 Examples of $3^{\text {rd }}$ order central symmetrical pattern

Fig. 4 A $2^{\text {nd }}$-order circular symmetry pattern

Fig. 5 Hexagonal matching pattern

Fig. 6 Hexagonal Tessellation

Fig. 7 Six layout patterns used in simulation

Fig. 8 Two precisely-matched resistor pairs

TABLE I. SIMULATION RESULTS OF DIFFERENT LAYOUT PATTERNS 
Corresponding author: Chengming He, gehe@ silabs.com

7000 West William Cannon Drive,

Building 1, Suite 200

Austin, TX 78735

Phone: 512-532-5869 Fax: 512-428-1555 